\title{
Threat Degree Sorted of Multi-Protected-Target in Air Combat Platform
}

\author{
Bo Liu ${ }^{1}$, Haibao Xia ${ }^{2, a, *}$, Wenbiao Long ${ }^{2}$, Nan Wang ${ }^{2}$ \\ ${ }^{1}$ Science and Technology on Avionics Integration Laboratory, Shanghai 200233, China \\ ${ }^{2}$ Aeronautics and Astronautics Engineering College, Air Force Engineering University, Xi'an \\ 710038, China \\ ${ }^{a}$ Corresponding author: Haibao Xia.email:2597969739@qq.com
}

Keywords: air combat platform; multi-target attack; threat assessment; negative relational analysis

Abstract: This article makes comprehensive threat assessment that is aimed at multi-target facing multi-protected-object with multiple negative relational analysis method based on set multi-protected-object according to the situational relation between multi-combat-unit of air combat platform. Moreover, it shows the feasibility of the methods provided in this article through simulation confirmation.

\section{Introduction}

With the rapid development of information, air combat platform faces the challenge of multi-task, Aircraft radar needs to make threat assessment on these targets while discovering quantities of battlefield targets.

Adopting the conservational gray correlation analysis method, Document [1-2] selecting positive ideal sequence (ideal target's evaluation numerical, row vector) as a reference sequence, weighted associate degree, using it as a comprehensive evaluation index for assessment sequence target. Based on conservational gray correlation analysis method, Document [3-4] utilizing the correlation between positive ideal program and negative ideal program, obtaining new objective function using least squares that solve the square averaging of positive and negative correlation, adopt this objective function as comprehensive evaluation index of the value program. However these documents just research threat assessment in multi-target, without taking the influence target threat level makes on the integrity of multi-protected-objects into consideration.

This paper makes comprehensive threat assessment on multi-protected-objects which is aimed at multi-targets according to the relationship between multi-combat-units of air combat platform and using multiple negative relational analysis method based on set multi-protected-objects. Simulation result shows that the evaluation algorithm in this paper is consistent with the actual situation.

\section{Targets threat assessment on multi-protected-objects modeling}

Airborne radar get target data $(R, \theta, \alpha), \mathrm{R} 、 \theta 、 \alpha$ respectively are relative distance, azimuth angle, pitch angle, and target radial velocity $V_{r}$. When doing the evaluation threat not for this aircraft, it cannot be used directly the airborne radar measurement data, and need to convert measured parameter. Example pole coordinate system converting to rectangular coordinate system as: 


$$
\left\{\begin{array}{c}
x^{\prime}=R \cos \alpha \cos \theta \\
y^{\prime}=R \cos \alpha \sin \theta \\
z^{\prime}=R \sin \alpha
\end{array}\right.
$$

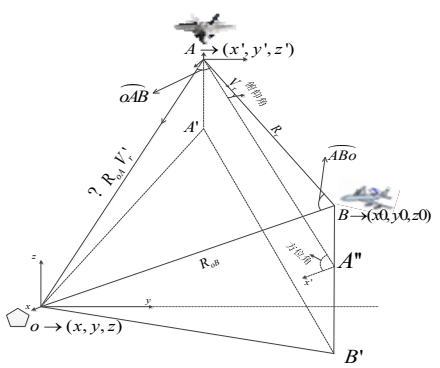

Figure 1. Parameter converting diagrammatic drawing

The relative distance between object and target:

$$
R_{o A}=\sqrt{\left(x-x^{\prime}\right)^{2}+\left(y-y^{\prime}\right)^{2}+\left(z-z^{\prime}\right)^{2}}
$$

The relative velocity between object and target:

$$
V_{r}^{\prime}=V_{r} * \cos \widehat{O A B}
$$

In which, $V_{r}^{\prime}$ is relative velocity between object and target,

$$
\cos \overparen{O A B}=\frac{R_{r}^{2}+R_{o A}^{2}-R_{o B}^{2}}{2 R_{r} R_{o A}}
$$

According to Gray incidence model ${ }^{[5]}$ :

$$
r_{j}(k)=\frac{a+b \rho}{\Delta_{j}(k)+b \rho}
$$

In which, $\Delta_{j}(k)=\left|b_{k j}^{\prime}-b_{k i}^{\prime}\right|, a=\min _{1 \leq k \leq n} \min _{1 \leq j \leq m} \Delta_{j}(k), b=\max _{1 \leq k \leq n} \max _{1 \leq j \leq m} \Delta_{j}(k), \rho$ is threat coefficient, interval $[0,1]$.

According to the target data offered by airborne radar, factors for influencing air-attack target threat level:

1) Flight altitude; the higher the flight altitude, the lower the threat level.

2) Relative distance; the farther the relative distance, the lower the threat level.

3) The number of formation; the less the number of the formation, the lower the threat level.

4) IFF; three kinds of IFF: enemy, unknown, friend, it is defined as the value: 1 enemy, 2 unknown, 3, friend. The less the IFF number, the lower the threat level.

5) Radial velocity; the greater the radial velocity, the higher the threat level.

6) Target type; it is defined different types of targets correspond to different numerical value.

7) The smaller the number, the lower the threat level.

It requires dealing with dimension owing to that each influence factor of threat level assessment has different dimension which makes the incapability of modeling. Suppose Early Warning Aircraft need to protect $\mathrm{K}$ targets, at the same time there are $\mathrm{n}$ targets, each one attribute has $\mathrm{m}$, attribute value bij represent attribute pj of target si, defining matrix $B=($ bij $) n^{*} m$ as judgment matrix. To make every attribute comparable, we do non-dimensional treatment for the matrix.

For the benefit attributes, its property value the bigger the better: 


$$
b_{i j}^{\prime}=b_{i j}-b_{\min }^{j} / b_{\max }^{j}-b_{\min }^{j} ; \quad b_{\max }^{j}=\max _{1 \leq i \leq n} b_{i j} ; \quad b_{\min }^{j}=\min _{1 \leq i \leq n} b_{i j}
$$

For the cost attributes, its property value the smaller the better:

$$
b_{i j}^{\prime}=b_{\max }^{j}-b_{i j} / b_{\max }^{j}-b_{\min }^{j} ; \quad b_{\max }^{j}=\max _{1 \leq i \leq n} b_{i j} ; \quad b_{\min }^{j}=\min _{1 \leq i \leq n} b_{i j}
$$

So the non-dimensional treatment attribute matrix of assessment method in $\mathrm{n}$ targets is:

$$
B^{\prime}=\left(b_{i j}^{\prime}\right)_{n^{*} m}
$$

\section{Case analysis}

Assuming that the aircraft platform preforming a particular task, need to protect a target from attack, assuming the protected target as a point target. Suppose when the Early Warning Aircraft is in a certain position, its radar find five groups of target, and fly towards the Early Warning Aircraft. After airborne sensors detecting has got the target data, the data is shown in table2, setting coordinates $(0,0,0)$, protected-targets coordinate $(500,-100,-9)$, unit: kilometer.

\subsection{The airplane multi-targets threat assessment}

Table 1 The meaning of common symbols

\begin{tabular}{|c|c|c|c|}
\hline symbol & implication & symbol & implication \\
\hline $\boldsymbol{H}$ & Flight altitude & $\boldsymbol{V}_{r}$ & Radial velocity \\
\hline $\boldsymbol{R}$ & Relative distance & $\mathbf{T}$ & Target type \\
\hline Num & The number of formation & $\mathbf{E}$ & Comprehensive evaluation \\
\hline IFF & Identification Friend-or-Foe & & \\
\hline
\end{tabular}

Table 2 The targets attribute value of this aircraft

\begin{tabular}{|l|c|c|c|c|c|c|}
\hline & $\boldsymbol{H}$ & $\boldsymbol{R}$ & Num & IFF & $\boldsymbol{V}_{r}$ & $\mathbf{T}$ \\
\hline group 1 & 4 & 269 & 5 & 1 & 300 & 3 \\
\hline group 2 & 1 & 501 & 4 & 3 & 350 & 2 \\
\hline group 3 & 0.8 & 149 & 6 & 2 & 200 & 5 \\
\hline group 4 & 5 & 141 & 20 & 1 & 800 & 1 \\
\hline group 5 & 2 & 102 & 23 & 2 & 600 & 4 \\
\hline
\end{tabular}

Table 3 The non-dimensional treatment judgment matrix of this aircraft

\begin{tabular}{|c|c|c|c|c|c|c|}
\hline & $\boldsymbol{H}$ & $\boldsymbol{R}$ & Num & IFF & $\boldsymbol{V}_{r}$ & $\mathbf{T}$ \\
\hline group 1 & 0.36 & 0.6666 & 0.2174 & 1 & 0.375 & 0.6 \\
\hline group 2 & 0.96 & 0.2033 & 0.1739 & 0.3333 & 0.4375 & 0.8 \\
\hline group 3 & 1 & 0.9045 & 0.2609 & 0.6667 & 0.25 & 0.2 \\
\hline group 4 & 0.16 & 0.9213 & 0.8696 & 1 & 1 & 1 \\
\hline group 5 & 0.76 & 1 & 1 & 0.6667 & 0.75 & 0.4 \\
\hline
\end{tabular}


Table 4 The correlation coefficient matrix of this aircraft

\begin{tabular}{|c|c|c|c|c|c|c|}
\hline & $\boldsymbol{H}$ & $\boldsymbol{R}$ & Num & IFF & $\boldsymbol{V}_{r}$ & T \\
\hline group 1 & 0.3962 & 0.5575 & 0.3492 & 1 & 0.4019 & 0.5122 \\
\hline group 2 & 1 & 0.3569 & 0.3482 & 0.4013 & 0.4456 & 0.7241 \\
\hline group 3 & 1 & 0.8147 & 0.3623 & 0.5575 & 0.359 & 0.3443 \\
\hline group 4 & 0.3333 & 0.8421 & 0.7630 & 1 & 1 & 1 \\
\hline group 5 & 0.6364 & 1 & 1 & 0.5575 & 0.6269 & 0.4118 \\
\hline
\end{tabular}

As far as Early Warning Aircraft concerned, five groups target comprehensive threat evaluation coefficient are: $0.5496,0.5478,0.5919,0.8180$, and 0.7070. Target assessment is group4>group5>group3>group1>group2.

\subsection{The aircraft and protected object whole (APOW) multi-targets threat assessment}

Table 5 The targets attribute value of object1

\begin{tabular}{|l|c|c|c|c|c|c|c|}
\hline & $\boldsymbol{H}$ & $\boldsymbol{R}$ & Num & IFF & $\boldsymbol{V}_{r}$ & $\mathbf{T}$ & $\mathbf{E}$ \\
\hline group 1 & 4 & 531 & 5 & 1 & 99 & 3 & 0.5739 \\
\hline group 2 & 1 & 537 & 4 & 2 & 182 & 2 & 0.5752 \\
\hline group 3 & 0.8 & 465 & 6 & 2 & -30 & 5 & 0.6295 \\
\hline group 4 & 5 & 632 & 20 & 1 & 714 & 1 & 0.8557 \\
\hline group 5 & 2 & 557 & 23 & 2 & 320 & 4 & 0.7234 \\
\hline
\end{tabular}

Table 6 The non-dimensional treatment judgment matrix of object1

\begin{tabular}{|c|c|c|c|c|c|c|c|}
\hline & $\boldsymbol{H}$ & $\boldsymbol{R}$ & Num & $\mathbf{I F F}$ & $\boldsymbol{V}_{r}$ & $\mathbf{T}$ & $\mathbf{E}$ \\
\hline group 1 & 0.36 & 0.8955 & 0.2174 & 1 & 0.1392 & 0.6 & 0.6707 \\
\hline group 2 & 0.96 & 0.8861 & 0.1739 & 0.3333 & 0.2547 & 0.8 & 0.6722 \\
\hline group 3 & 1 & 1 & 0.2609 & 0.6667 & -0.0420 & 0.2 & 0.7356 \\
\hline group 4 & 0.16 & 0.7359 & 0.8696 & 1 & 1 & 1 & 1 \\
\hline group 5 & 0.76 & 0.8550 & 1 & 0.6667 & 0.4487 & 0.4 & 0.8453 \\
\hline
\end{tabular}

Table 7 The correlation coefficient matrix of object 1

\begin{tabular}{|c|c|c|c|c|c|c|c|}
\hline & $\boldsymbol{H}$ & $\boldsymbol{R}$ & Num & IFF & $\boldsymbol{V}_{r}$ & $\mathbf{T}$ & $\mathbf{E}$ \\
\hline group 1 & 0.4487 & 0.8330 & 0.3997 & 1 & 0.3770 & 0.5657 & 0.6127 \\
\hline group 2 & 1 & 0.8758 & 0.3986 & 0.4540 & 0.4248 & 0.7650 & 0.6442 \\
\hline group 3 & 1 & 1 & 0.4134 & 0.6098 & 0.3333 & 0.3944 & 0.6634 \\
\hline group 4 & 0.3828 & 0.6636 & 0.7998 & 1 & 1 & 1 & 1 \\
\hline group 5 & 0.6846 & 0.7823 & 1 & 0.6098 & 0.4859 & 0.4648 & 0.7711 \\
\hline
\end{tabular}

As far as The APOW concerned, five groups target comprehensive threat evaluation coefficient are: $0.5932,0.6281,0.6596,0.8252$, and 0.6921. Target assessment is group4>group5>group3>group2>group1. The obvious contrast in the table above shows that the assessment results between this aircraft and multi-protected-objects have differences to some degree. The target 2 has the lowest threat level when assessing on this aircraft due to that it has much longer distance for this aircraft. However, as to the integrity of protected objects, the relative distance between target 1 and this aircraft is small while radial velocity of target 2 is bigger than target 1 . 
Therefore, target 2 is ranked before target 1 when aiming at making threat assessment on protected objects.

\section{Conclusions}

This article makes research on the sorting issue aiming at making thereat assessment on target aircraft by the integrity of protected objects starting from carrying out combat missions on air combat platform. The method provided in this article has guiding significance on threat level assessment on multi-targets and multi-objects, and is much more useful for carrying out air combat missions.

\section{Acknowledgements}

This research was funded by the Science and Technology on Avionics Integration Laboratory \& Aeronautical Science Fund (20155596024); This research was funded by the Science and Technology on Avionics Integration Laboratory \& Aeronautical Science Fund (20145596025).

\section{References}

[1] YU Jun-shan,ZHANG Tao,CAO Ning, et al. Priority Evaluation of Air Threat Based on Gray Correlation Analysis [J]. Fire Control \& Command Control, 2004, 8 (29):68-71 (in Chinese).

[2] Liu Jian. Study of Threat Evaluation Model of Air Targets Based on Grey Incidence Decision-making [J]. Tactical Missile Technology, 2010, (6):49-52 (in Chinese).

[3] Ma Lin, Song Guibao, Ji Lichao, et al. Evaluation of Target Threat Based on Least Square Gray Correlation Analysis Method [J]. Tactical Missile Technology, 2010, (1):28-31 (in Chinese).

[4] WANG Wei, SHI Hong-quan, ZHOU Ai-jun, et al. Aerial Target Threat Assessment of Formation Collaborative Combat Under Uncertain Environment [J]. Command Control \& Simulation, 2014, (4):12-15 (in Chinese).

[5] SUN Hai-yong, CHEN Yang-ye, HAN Hui-gang. Assessing and Ranking of Air-Raid Target Threat Based on Grey Clustering [J]. Journal of Air Force Radar Academy, 2011, (5):355-357,361 (in Chinese) 